\title{
The Voyage of Giovanni da Verrazzano A Newly Discovered Manuscript
}

\author{
by Dionysios Hatzopoulos and Richard Virr
}

The expedition of Giovanni da Verrazzano in 1524 under the auspices of Francis I of France was one of the important early voyages of discovery. The text of Verrazzano's report has been known to twentieth-century bistorians in five versions: four manuscripts and a printed text. However, nineteenth-century researchers knew of the existence of another manuscript text bound into the Verrazzano family copy of Ramusio's Delle navigatione et viaggi raccolta. This manuscript was recently identified in the boldings of the Department of Rare Books and Special Collections, McGill University Libraries, and is bere described and printed for the first time.

L'expédition de Giovanni da Verrazzano en 1524 sous les auspices de François $I^{\text {er }}$ de France fut l'un des premiers grands voyages d'exploration. Le récit de Verrazzano est connu des bistoriens du $X X^{*}$ siècle en cinq versions: quatre manuscrits et un texte imprimé. Toutefois, les cbercheurs du $X I X '$ siècle étaient au courant de l'existence d'un autre manuscrit incorporé à l'exemplaire de l'ouvrage de Ramusio Delle navigatione et viaggi raccolta appartenant à la famille Verrazzano. Ce manuscrit a récemment été découvert dans le fonds du département des livres rares et des collections spéciales des bibliothèques de l'Université McGill et il est décrit et reproduit ici pour la première fois.

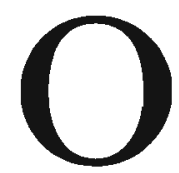
n 17 January 1524, the French caravel Daupbine sailed west from an island in the Madeira group in the Atlantic Ocean off the coast of Portugal in search of the long sought western passage to the shores of Cathay. It was a small ship of one hundred tons with a crew of fifty men. ' Commissioned by Francis I, King of France, the Dauphine was destined to explore the coast of North America. While Antoine de Conflans, an able navigator, was captain of the vessel, the Florentine Giovanni da Verrazzano was the leader of the expedition and the person responsible for carrying out the royal commission. After fifty days crossing the Atlantic, landfall was made in a place which has not been identified with precision. However, it has been suggested that the expedition may have reached the area close to where the North and South Carolina boundary reaches the ocean. ${ }^{2}$

Having reached land, the Dauphine sailed north along the Atlantic coast until it reached the Cape Breton-Newfoundland area. The coast and its inhabitants were observed and described by Verrazzano and his account was the first to do so, containing important ethnological information. ${ }^{3}$ Sailing near Newfoundland, known to Verrazzano as "Bacalaia," a name given by the Portuguese who fished in the area annually, the ship turned eastward and set sail for Normandy.

Arrived in Dieppe, Verrazzano wrote a letter-report to Francis I summarizing the information collected during the voyage. This letter dated 8 July 1524 was probably written in Italian, Verrazzano's mother tongue, and was then to be translated into French. The Italian version of the letter was addressed to either Leonardo Tedaldi or to Thomaso Sartini, merchants in Lyons. The explorer asked them to forward the letter to Bonacorso Ruscellay, a banker in Rome. All three men were members of prominent Florentine families and backers of Verrazzano. ${ }^{4}$

While the final report in French, if there ever was one, has never been found, a number 
TER ZO VOL V M E

\title{
DELLE NAVIGATIONI ET VIAGGI
}

\author{
RACCOLTO GIA DA M. GIO.BATTISTA RAMVSIO
}

NEL CVALE SI CONTENGONO

LeNauigationi al Mondo Nuouo, a gli Antichi incognito, fatte da Don Chriftoforo Colombo Genouefe, che fu il Primo à fcoprirloài R E CA TH O L I CI, detto horal'Indic occidentali, con gl' acquifti facti da lui, \& accrefciuti poi da Fernando

Cortefe, da Francelco Pizzarro, \& altri valorofi Capitani, in diuerfe parti delle dettelndie, in nome di C A R L O v. IMP. Con lo fcoprire la gran Cirtì di Temiftitan nel Mexico, doue hora è detrola nv O VA SPA g Na, \& lagran Prouinciadel Perù, Il grandiffimo fume Maragnon, Et altre Citrì, Regni, \& Prouincie.

Le Nauigationi fatte dipoi alle dette Indie, poste nella parte reryo Maefro T ramontana, dette borala NVOYA FRANCTA, foperte al RE CHRISTIANISS. La prima volia da Bertoni (E) Normandi, Et dipoi da Giouranni da Verrazzano Fiorentino, w dal Capitano Lacques Carthier.

Si come ff jegge nelle diverfe Relationi, tradcite dal Ramufio di Lingua Spagnuola \& Francefe nella noftra, \& raccolte in quefto volume.

Con Tasole di Geographia, che dimostrano il fro di dinerfe Ifole, Cirtà, of Paffs. Et Figure diuerfe di Piante, of alirc cooc a noi incognite.

Et con L'Induce copsodyssimo di turte le cofe pix notabili in effo contenuse.

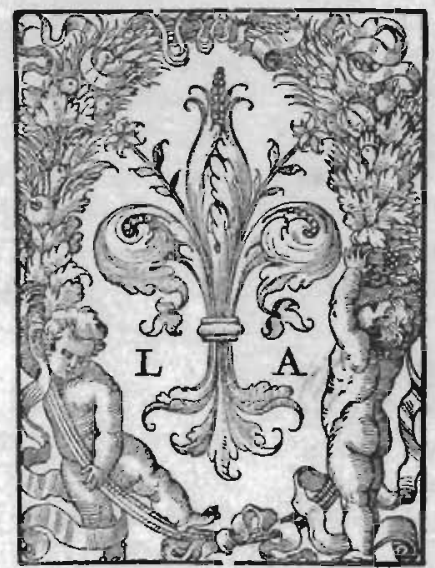

IN VENETIA NELLA ST $\mathcal{M}$ MPEIA DE'GIVNTI:

LINNO M D LXV.

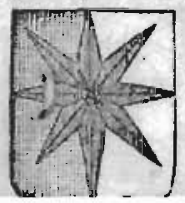

Figure 1. Title page of the McGill copy of Ramusio with the Verrazzano arms at the bottom of the page. 
of versions of the Italian original survive. 'The known extant versions which carry the designations given to them by Alessandro Bacchiani are as follows:

\section{$\mathrm{R}$, the Cèllere version, now Morgan Ms. MA. 776, a manuscript of the sixteenth century, a complete text published first by Alessandro Bacchiani and in a modern edition with an English translation by Lawrence Wroth.' \\ $F$, the Magliabechian version in the National Library of Florence, again a complete text presumably a man- uscript of the sixteenth century, published by Giuseppe Arcangeli in 1853 and Guglielmo Berchet in $1892 .^{\circ}$}

$\mathrm{Rm}$, the Ramusio version in volume three of Delle navigationi et viaggi raccolta, Venice, 1565, leaves $420-422$, this contains only the first part of the letter and omits the cosmographical section.'

$C$, the Cimento fragment, in the Accademia del Cimento, bears the stamp of Ramusio and contains only the latter part of the cosmographical section. It was published by Alessandro Bacchiani. ${ }^{8}$

$\mathrm{V}$, the Vatican version MS. Ottoboniano 2202, again probably a sixteenth century manuscript, described by Alessandro Bacchiani.

Of the five versions, only $\mathrm{R}, \mathrm{F}$ and $\mathrm{V}$ provide a complete text of the account of the voyage. Ramusio published only the first part of the text, i.e. the ethnographical portion, and the Cimento fragment (C) contains only the last two paragraphs of the cosmographical part.

In addition to these versions of the text available for study today, nineteenth century researchers of Verrazzano's life were aware of the existence of another manuscript of the report to Francis I, bound into the Verrazzano family's copy of Ramusio's work. The first to mention the existence of this manuscript was G.W. Greene in an article in the October 1837 issue of the North American Review. ${ }^{10}$ Shortly after the death of the last member of the Verrazzano family, Greene visited the family library, looking for information on the explorer. There he was informed by the person who had examined the volumes contained in the library previous to their being sold that "all...that was found in it relative to Giovanni [da Verrazzano] was a manuscript bound up in the family copy of Ramusio, and a few loose papers." Subsequently, Greene stated that he presumed that the document "was nothing more than a copy of the ... cosmographical description, or perhaps of the whole letter, from the Magliabechian manuscript."11

Ramusio's three volume work had been sold sometime before 1837 to a Captain Napier, R.N. This was very probably Captain Henry Edward Napier (1789-1853), a well known collector. ${ }^{12}$ Greene expressed in his article the wish that Napier would describe the manuscript, but nothing came of this. The manuscript was mentioned again in 1853 by Giuseppe Arcangeli, when he published the Magliabechian version (F). ${ }^{13}$ When in 1871 , J.C. Brevoort mentioned Greene's visit to the Verrazzano library as well as his hypothesis about the manuscript's contents and value, it had again changed hands. ${ }^{1 / 4}$ Alessandro Bacchiani mentioned the manuscript again in 1909 . He said that it had been acquired by Napier before 1846 (Greene had placed this acquisition before 1837); but, in any case by 1909 , the manuscript was considered lost and its value still unknown. ${ }^{\text {is }}$

In fact, sometime following Napier's death in 1853, Ramusio's work was acquired by the Canadian industrialist Peter Redpath (1821-1894), a governor of McGill College, Montreal and a benefactor of the Library. In 1877, Redpath gave the Ramusio to the McGill College Library. ${ }^{16}$ The set is composed of three volumes: the first dates from 1563, the second from 1583 and the third from 1565. Each volume has the star-shaped coat of arms of the Verrazzano family pasted in the centre of the lower margin of the title page (Figure 1). In the third volume, the manuscript is bound in between ff. 422 and 423, that is, following the section in which Ramusio printed the first part of the Verrazzano text. 


\section{The Voyage of Giovanni da Verrazzano}

The manuscript, from now on called "McGill" for purposes of brevity, is composed of eight water-marked leaves, having text on both sides except for the verso of the fourth leaf and the eighth leaf which are blank. The water-mark has not been identified; it consists of the Medici arms surmounted by a royal crown. ${ }^{17}$ The text is written in black ink with a wide pen in the clear round hand of the midseventeenth century that was used in most of Italy at that time. The text is divided into two parts, each one beginning with an elaborate calligraphic initial - " $R$ " for the first section and " $G$ " for the second. The ink, profusely used in the initials, has now acquired the familiar rusty brown colour and its acids have begun to eat away the paper.

The first section contains the "parte cosmographia" omitted by Ramusio. The McGill text is closer to that of the Magliabechian (F) version than it is to the Cellere $(\mathrm{R})$ version, without, however, any indication that it was based on the former. In fact, all extant versions seem to descend from different copies of the original. "McGill" contains words which are missing or spelled differently in the other extant versions. In one case, $\mathrm{R}$ omits a long phrase which $\mathrm{F}$ and $\mathrm{McG}$ ill contain: “...propinqui all equatore più all' occidente participando più al settentrione giusta la detta linea meridionale continovando il lido per fino in gradi 21 non trovando termine..." (lines 63-66). ${ }^{18}$

On the other hand, "McGill" contains a number of errors found in $F$ and noted by Bacchiani which probably indicate that the two texts are closely related. Thus, the incomprehensible "...se lo equestre di detta terra in parte corrisponde..." of $\mathrm{F}$ is repeated verbatim in "McGill;" in $\mathrm{R}$ this appears as "...se il sito terrestre di detta terra in parte corrisponde..." Also, the McGill scribe was unable to read "Rossia" and wrote "orsia." But then while F has "...promontorio di capo di Buona Speranza...", "McGill" has the erroneous "...promontorio dicano di Buona Speranza..." Furthermore, while $\mathrm{R}$ has the comprehensible "...tutta questa terra...non giugnendo a la Asia ne a la Africa...," "McGill" and $\mathrm{F}$ have "...tutta questa nova terra...congiungendo all'Asia et Africa..." "Cimbri" in R becomes "Cimbi" in McGill. "A li antiqui noto" in $\mathrm{R}$ becomes the incomprehensible "all' antiquinozio" in "McGill." But then "McGill" says "...in Tatitudine gradi 32 dall' equatore..." while R says incorrectly "...in altitudine gradi xxxii da l'equatore..." All the extant versions contain numerous spelling and grammatical mistakes, and they all contain many variations in the equations for longitude and latitude.

The second part of the manuscript, written by the same hand, has for a title "Capitolo tratto dal Libro in lingua Fiamminga intitolato Mondo Nuovo, o vero, Descrizione Dell Indie Occidentali Raccolta di Molte Scritture e Note di diverse Nazioni da Gio. De Laet. Stampato in Leiden da Isaac Eltevier, l'Anno 1625. Con privilegio di 12. Anni dalli SSri. Stati Generali. Descrizione dell' indie Occidentali, Libro III. Virginia, Capitolo I. Foglio 74 - Il Viaggio di Gio. da Verrazzano." This is an Italian translation of Joannes de Laet's original Dutch version of Nieuive Wereldt of te Beschrijvinghe van West-Indien... published in 1625. A second edition, much revised, appeared in 1630. A Latin translation of this second Dutch edition was published by the Elzevirs in 1633: Nowus Orbis seu Descriptionis Indiae Occidentalis, and a French translation, $L^{\prime}$ bistoire du Nouveau Monde..., followed in 1642. ${ }^{19}$ This extract from De Laet, translated, as it is, from the Dutch first edition, suggests that the McGill manuscript should be dated to the period between 1625 when the text was first published and 1633 when the Latin translation appeared. It would seem probable that if the Latin version or the French (1642) had been available, the translation into Italian would have been made from one of them rather than from the Dutch. Furthermore, the McGill text follows neither the Latin nor the French translation, both made from the second edition of De Laet's text. All of this suggests very strongly that the McGill text should be dated in the first half of the seventeenth century.

The McGill text of Verrazzano's letter adds significantly to the available versions. Its provenance - commissioned by a member of the Verrazzano family to complete Ramusio's text - gives it additional importance. Of the extant versions, only the Vatican text (V) has not been published. Furthermore, the McGill text contains an important phrase as does $F$ (lines 
The Voyage of Giovanni da Verrazzano
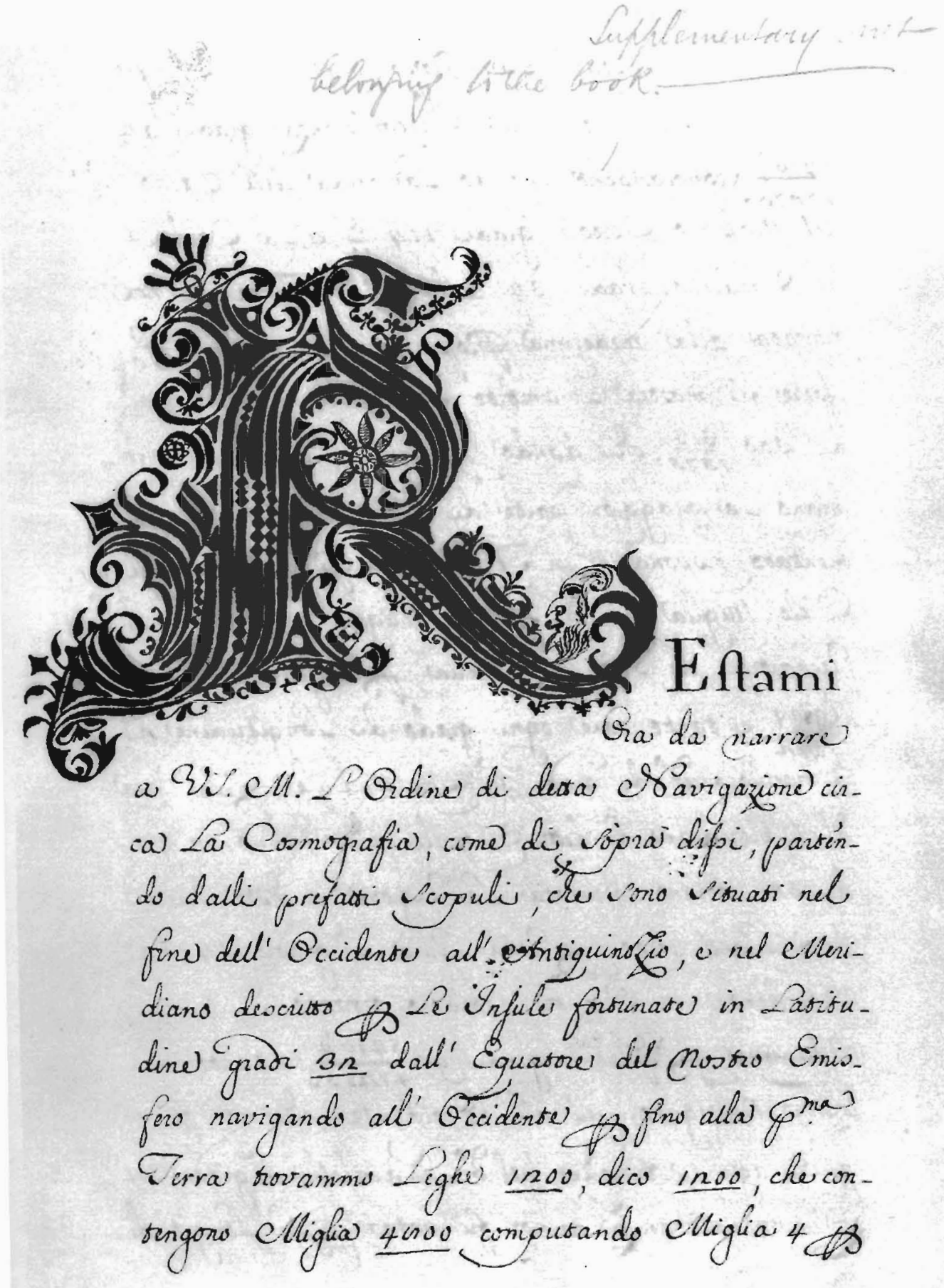
- cgar Secondo Puo ellavisiono delli efavilery

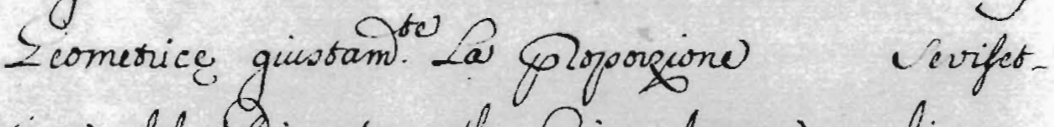
rimal del Oiametio alla Circorferenza gradi gn $\frac{54164}{47 n>33}$ conciosiachs epends Ia cordal dell' erico del mapimo Cireolo gradi $114 \frac{6}{11}$, o La Corda of Sanacillo gradie 34 delko 12 Perra da noi trovara alla medefima gloporzine gradi $\left(5 \frac{n n 3}{450}\right.$

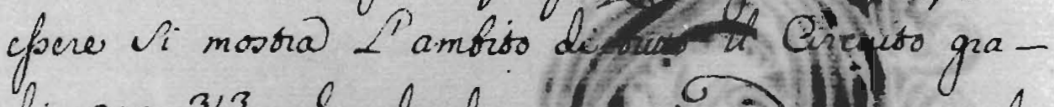

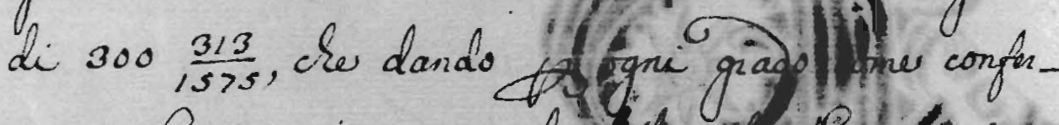

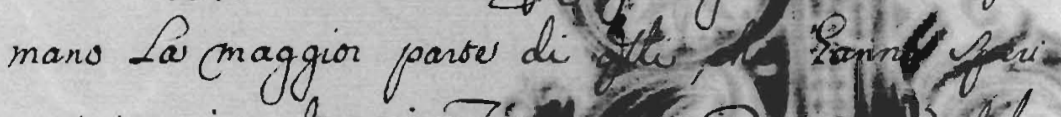

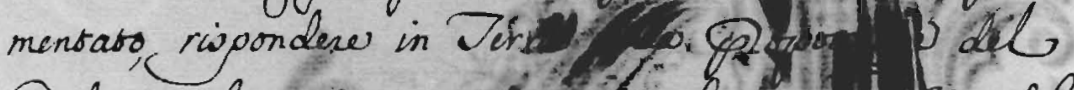

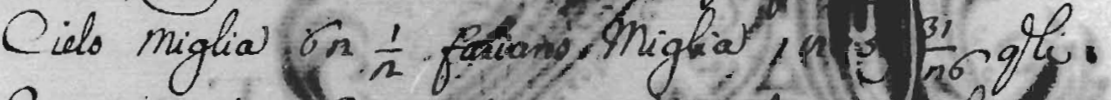

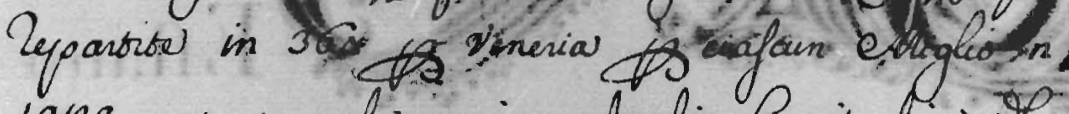

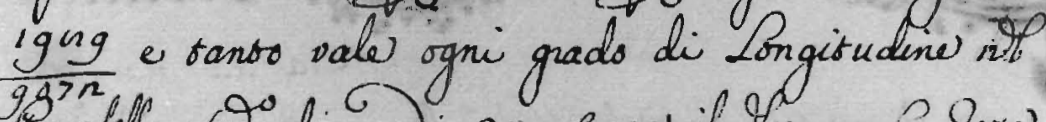

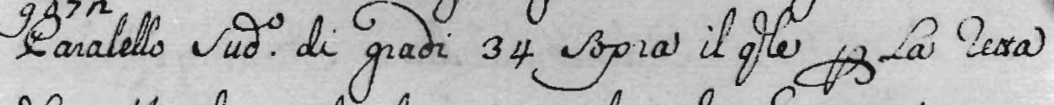
If Clleridians di desi Ceopuli, cher Sanno in gradi $3 n$ affiano calculara La Ragione in gasso, che Ls desser Legho inoo $\$ 8$ lewa, inea in gradi $34 \mathrm{da}$ Occidente in Qrientel affiamo trovato Adungues ofter a gradi gn $\frac{54164}{4>n 333}$ o santo af riamo navigato piu' all' Qccidente), che non fí cogniso agli contichi nel 20 Saralello digrad 34. (Guestar distanias a noi fui notas to La Longitudi- 
ne con vay Mumenti llavigando vinza Eclifi

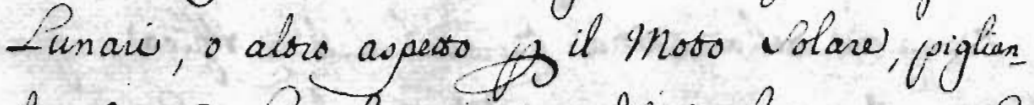

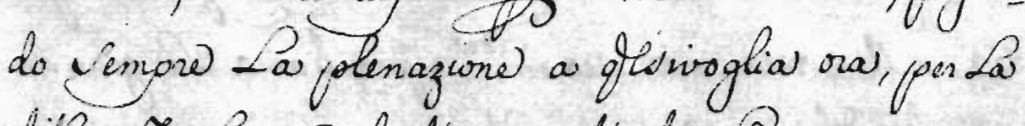
differenga gaceval dall' uns all' also Qizonte corren do a cravo Feomerice, ne era noro IPInterval. is dall' uno elleridians: alk' atso, come in un Lisherto tuso amplam. notaso insieme of crejamento of Cllare in offiriglias Clima a sgni temps, es ow, if ofte non inutice simo affial al errezel as ctravigans, vpens meglio La Terical conferinto a 31. . C.4. Mial insenzione eral di foenew in qora eravigazione alcathais all' cotreme Gienses Il' erfia pensando trovare sale impedimento di nusvar Derras, ofe ho trovasa, o ve gftche Ragione penja. vo qula srovare non senzal qualche fiegis di penesta. 10 all Qeeano Rientale, crocre vhimaro gora qpinisne di susci gr' CAntichi, i vtasa ondendo centam. of nofiro Qeeano Rientale de Indial uno ypere venza inberpofizione di Terra); gosto affermal Chiftotile, as = gumentando varie Simitisudini, La gite opinione

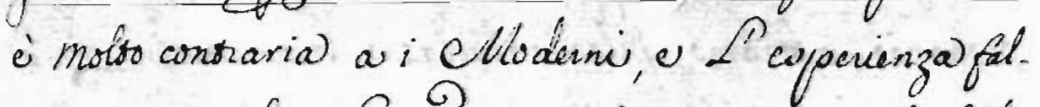
va, imperscche La Terra è stara trovara dia gith

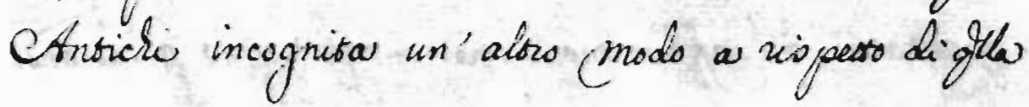




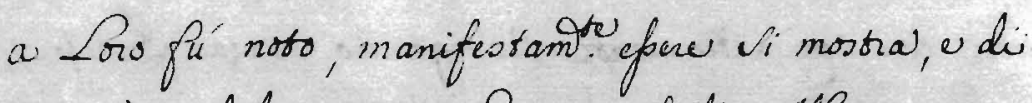
Maggiou della nostia Europa debl' effrical e qua.

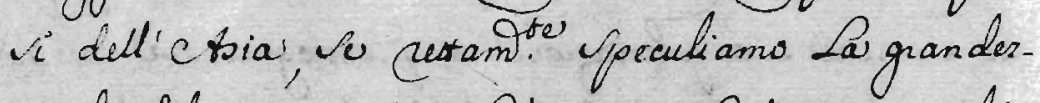

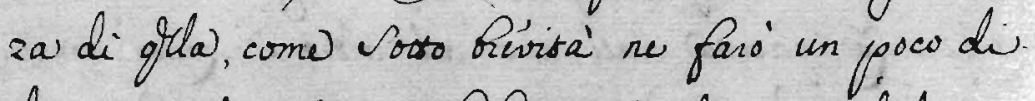
discorfo a 2. L.M. Equasore distanse dal meridiano dall Inoulo fortunate verfo Loccidents gradi no $\frac{32050}{47 n 001} 2$ Gpani verfo Denusto gradi 54 hanno navigato dove hanno sovato Jerra venza fine. Oonando poi al Petentrione giustal La deta Linea Mecidionaler correnda it Pido fofino in oro gradi

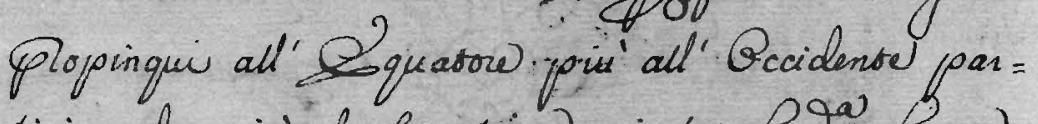
ticipando puir al Sestentrione guistar La da Limea Clevidionale consinovando it Lido fortino in gias nI non trovando termine gradi ung: $\frac{n g 70 q}{40>0 n}$ hanno Navigato, gli guenti con gradi no $\frac{3 n 060}{4 \sigma 3 n 1}$ fannogra. di $110 \frac{44 n 30}{4 \sigma>\ln 3}$, tanto hanno navigats of of:

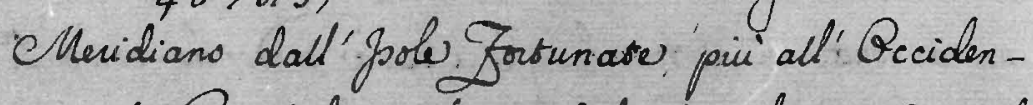
se, nel Paralello gradi nz oll' altitudine; gotal di. stanza das noi non e' statal Pperimentatas 4 non

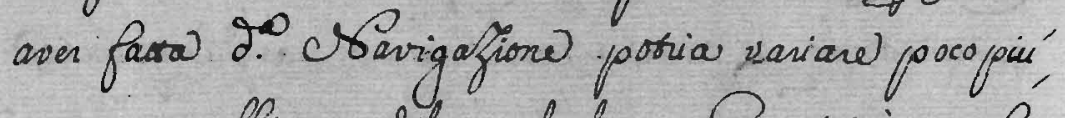

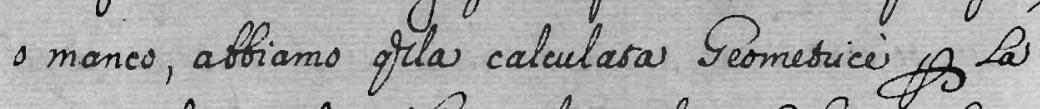
Norizia di Mols exaricatiey, che L hanno fre = quentata, gli affermano epere Legho 1600 giudicando 
The Voyage of Giovanni da Verrazzano

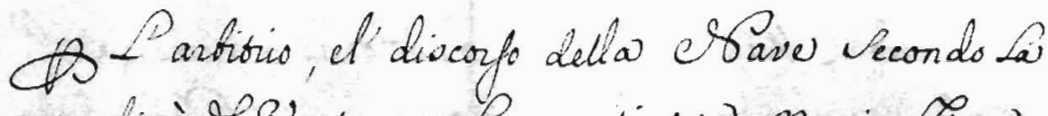
qualisai of 3 ento is 1 a continova Navigafione -pero in orere, ne daremo otimal centitudine. Walt altia parto noi in gisa nia ef Pavigazione fasal tS ordine di ina. S. Ul. olne i gradi gn, cherdal

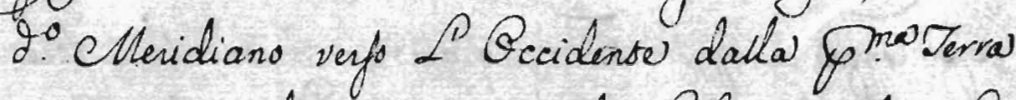
rovammo gradi 34, Navigando Leghe 300 , dico Leghe 300 infra Priense, o Sestentrione Peghe 400 quasi all' Oriente consinove, e Vito Tllal Terral Sia. mo pornuti infins a gradi so Laviando LaTas. ra), cher piu' sempo fa' sovorno li quortani quali

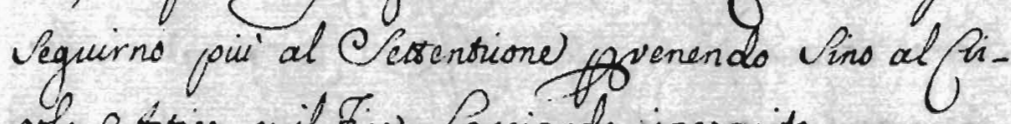
colo esirice, es it fine Paxiands incognito.

$(1)$

Uiunta adunque La Laritidine Astentuinale con La CMeridionale, videlices, gradi 54 con Li gradi 66 fanno gradi ino; che banto contiene di Lation. dine Leffrica con 2 Europa, parcer giugnen.

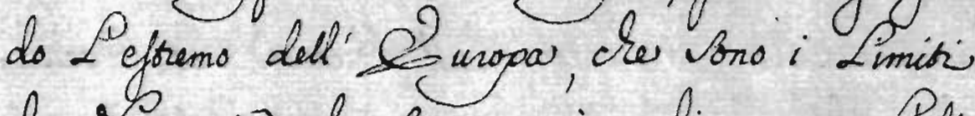
di cronvegia, cher fanno in gradi 21 con Lefore. mo dell' CAflica), che i il Cromonsonis, dicano di (Bung Spuanfa in gradi 35 fananno 10 b 200 , Ve iquester di gel Terra in parte corrispondes 
The Voyage of Giovanni da Verrazzano

al ido CUarisimo non i dubhi di granderza L Cffia ecceda, in sal forma toviamo al Glosio della Jerra molro maggione non hanns benuts gl'C tn = tichis a Cepugnant, o Cllastematici Sanno gtlar riopess all' etequa, via minima, th che per eoperienga Dopposito veggiamo, gीnto alt'ctere coyporales di paazio non meno La Terral, cher Pae qua porsedere giudichiomo come alla gejenzal, me. glio Spers, e con pui cagione esperimentare, es mo-

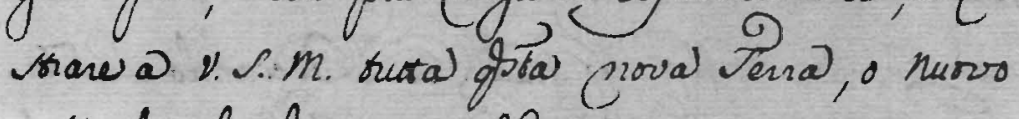
Cllondo, che disoral affiams narrato consine, in vieme conguingends all' ctfial, ot etffica), it cho Sappiamo certo, potria giugnere all' Europas con la Ofonvegia, e offia, che Sareffe fallo, secondo

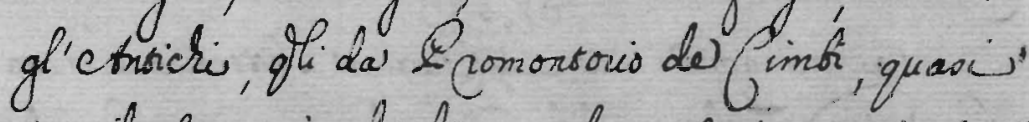

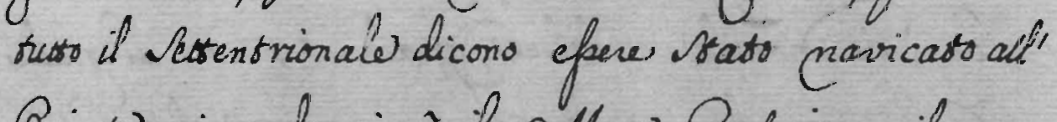
Giente, circuendo cical it ellare Cafpio, eril me. defimo affermans cestereffes adunque Sola inserchala La dw etlaw Vilicari dall Quents, er Qcciden. sales, os the dues ne chirdes I'uno, I'alsio, puckes otwe ai gran's 4 dell' Dquinsiale veyo "cru.

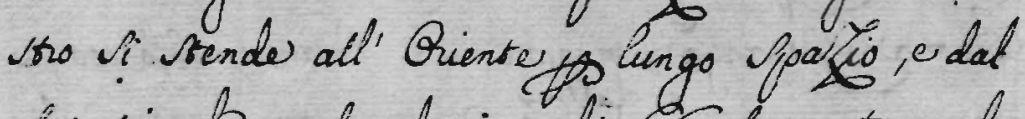
vertentionale papando i gradi 66 Seque trunando

16 
in viefo $P$ Qiento, giugrendo forno a gradi 70 Ppero con - ajus di v. S. EUl ne averemo in tieve

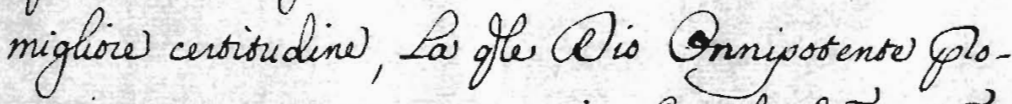
Spew a causal veggiamo strins pinet di gostal na Eojmografia, cher $\checkmark$ adempial La vacra tow dell' Evangels. Stella Orave Oelfina in Slorman

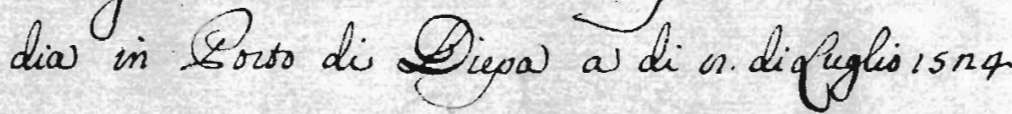

$$
\begin{aligned}
& \text { Humilis Servitor } \\
& \text { Joannes Verrazanus }
\end{aligned}
$$


Capitolo tratto dal Libro in lingua Fiamminga Intitolato $M$ ondo Nuovo, o vero Defcrizione. Dell'Indie Occidentali

Raccolta di molte Scritture, e Note di diverfe Nazioni da Gio. De Laet. Stampato In Ticiden da Iface Elteuier L'Anno 16 ns.

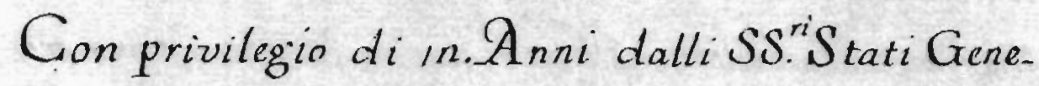
rali

Oefcriziö.dell' Indie Occidentali LibroIII. Virginia

Capitolo I. Foglio 74 -

Il Viaggio di Gio, da Verrazzano.

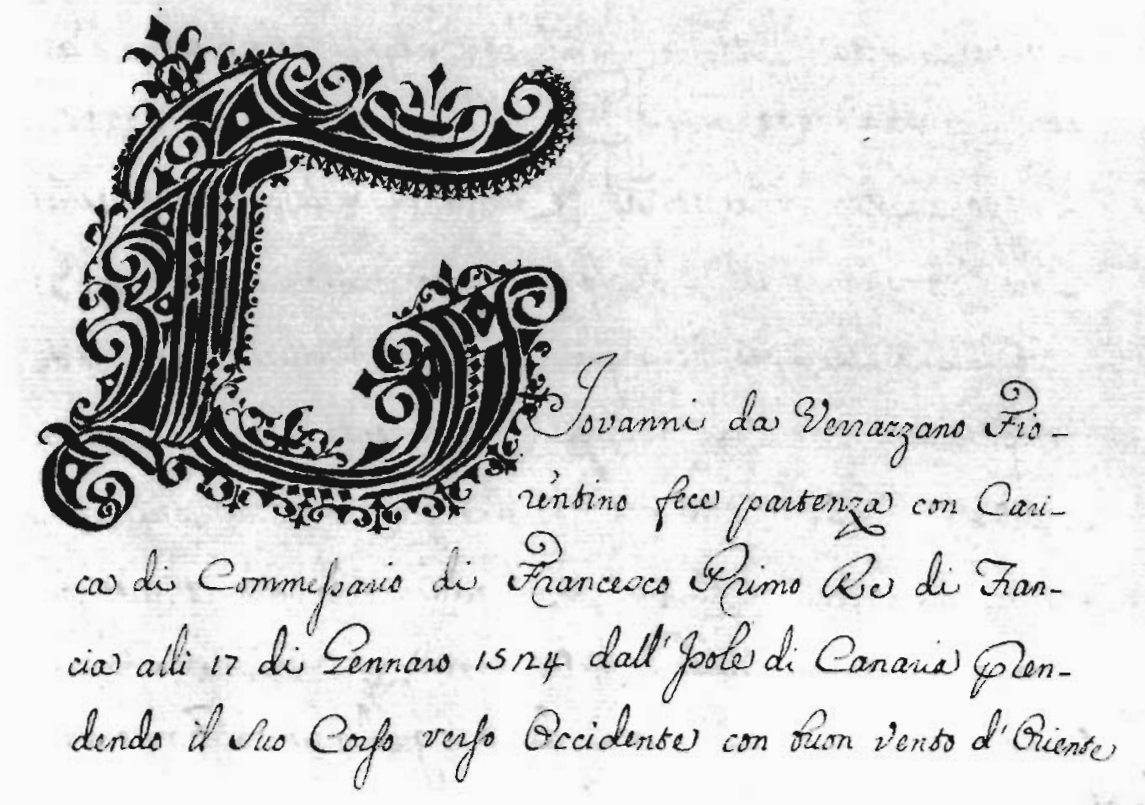


Enel tempo di gionni ns-feeers in circas soo-Leghor, di poi indirizzorno Po Coyfo d' Pecidenter a Pearentrione, dimods, cher doppos altri 125 giomi di Viaggis Scoperfero una Terral nuvar, cher giaceuar rasso, glepo Lacqua, ¿ts firochi, che colai vedevano, guidicorno, cherdorefve epere ariratas, es $i$ mipero vefo sitro; Yeggionno Sen so Legho alle Costo to trvare un Comods Conto, Mas non trovandolo in qu' etcquer verfo Ostro, trinarono vefo Camontana, dove medginamb non rovarino forti, Aické $A$ mepers verfo Le Costu, e mandando it Lo Poppans vegfo Terra, tovarons molsal gente alle Rivel, - vero Sengive, qfi prigliarono Lâ fuga', s qualcher vol. sas zestauano as guardare Spral Is Christioni con ias Bocca aperta, cllar finalm con Segni cersfigati, ches non li Saria fats alcun' incon to $p$ mopiono in fuon

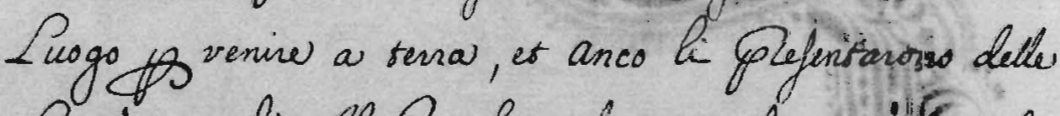

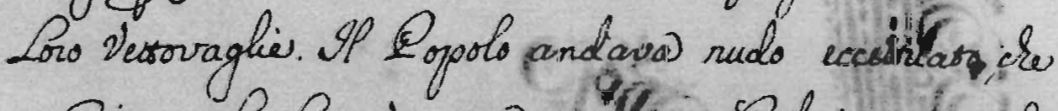

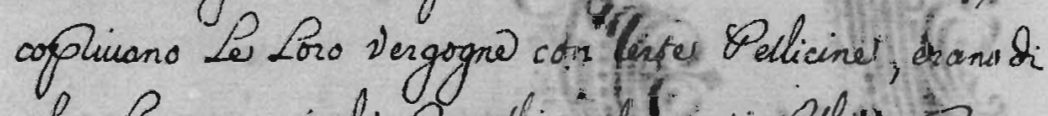

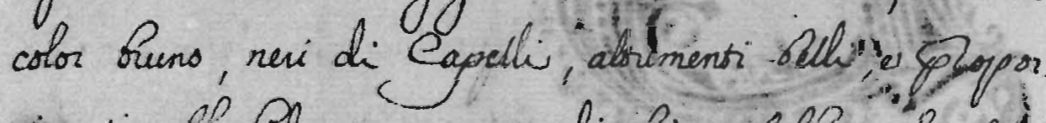
zionati, If Lido eras coperso di fina sabria, che vicfen deva in picesti eMuchi, a Paddicto is bello, et 'albo fon. do, aperso di Alberi, qosta Ferral, che glimal Eoper = fers eras Sopra - alsezzal di 34 gradi veys Tramontanes 
Ia Pinca, in una fiona, U Saluber aria, drovarons, che colla' Pfoter ta maggior parter regnavano venti di Cllaestro, el Ponente, con chiaro, es Lucido temps, eppocal pioggia, a $\curvearrowright$ dener enal un Lido apento non trovarons male a Starvi con le navi, trante Le coffe Sons Pibréchi Soghi. vi partirono di quivi senendo. i Lungo Lescosto, qli trovarono eftenderfi veyo Levan. to, es vedenono continovam. molti fuschi a terra, doppo twovarono il paeso ghenderfi verfo Gamontanas, il quale Ii moftraua molso piu bello, es ameno, calarono ta' a Terra, es rovarono La' genter Simites all'anteceden. ser, rederono molter vise, gte camminavano sopral ghi alberi, qfie al Pro parew potevans dar orioni puxi in cafo fupero State ben coltrivate, veleggiarons cosi, avanti, Secondo it Ló parene bén 100 - Leghs dove two. varons una belta (biviera), dove vi triaval di nuovo b Losta vafo Qiente, qhe plofeguirono cical so Legno, dove Noprions un' Iola, ofle aveua La forma sian golare, giacends so. Leghos dalla Terra gema prena) di Montagne, piene, es piepu di alberi, e tén abita. tos, qle nominarono elaudia, so Legho, pir avanti trovarono un Puon Gorto, dove sovorno Senter molso sicna, oratakte, gte avevano un $(B)$, ches era vefirio 


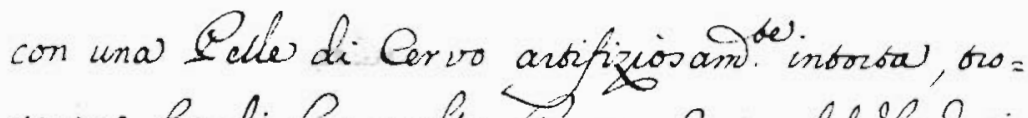

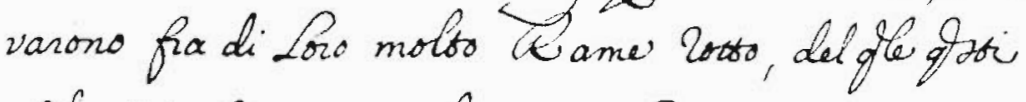
Valvatici faceuano molso conto Questa Berra, cra giacenter Sopra Lausezza di gradi $41 \frac{n}{3}$ e ciefsén deva fia Giente, e mezzo Giorno, $\angle a$ bocca df Sor. to giacu⿰冫 apertiv verfo. Conente, Langs mezzal Lga, ma di dentro Ni tirava tia Ouente, e Tramontana) in-Leghi, e La venuia puir Lango, e puir Lungo, es fá un grifo on Lango di no- Seghe, nel gle giacevano cingue piccole gooteser, qhi enano molso bêlle, e pusifere, nel meyo, vel circal dentio di qoso Sorso u

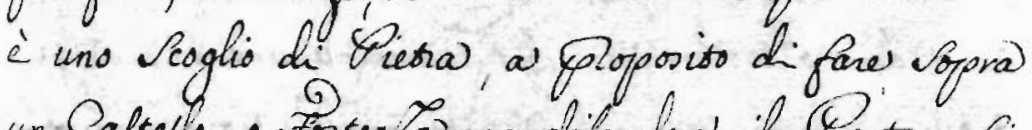

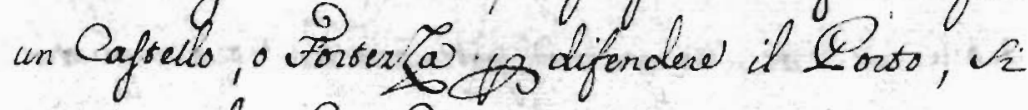
partions di la lis. ellaggio, confens avanti, applefo Le Pofte ten iso Legho trovando Laterra quari sum Sim, se non che cral un poco piui alta con alcune cllontagne, Lafortal î evitende. va da so. Legno veyfo Ouento, esdoppo di nuovo yelo Tamontana, rovanono cola $\hat{L}$ genter molso of. ferento dall' Antecedentel; Menore sicome ofles ms.

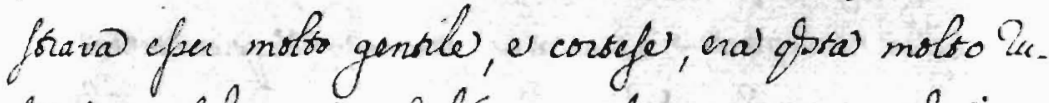
fica, e Salvatica; wiché to nebun mezzo con qdatiposeus aver aleun Commercio; Eanv velioi con pocter 


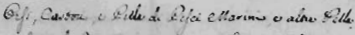

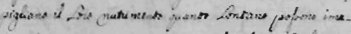

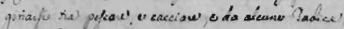

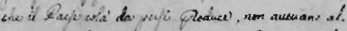

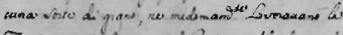

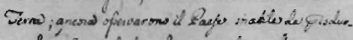

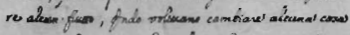

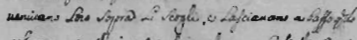

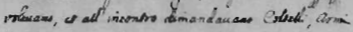

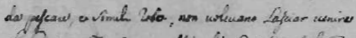

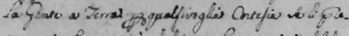

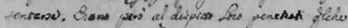

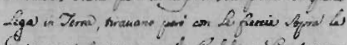

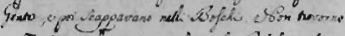

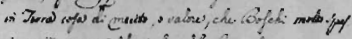

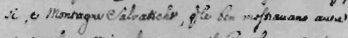

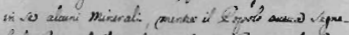

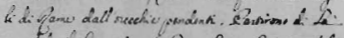

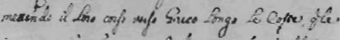

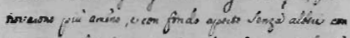

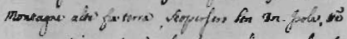

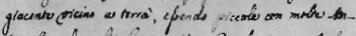

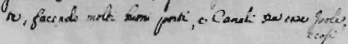


- cofi veleggiando avanti veefo Gues vennero dofpo un ve. leggiam di iso. qeghe vennero ath Terral, opaye awan. ti Scoperto dalli Bcutom, all'alrzzzal di so. Sradis apQupo zramontana), As Iineal d'onder cisolfero citornare

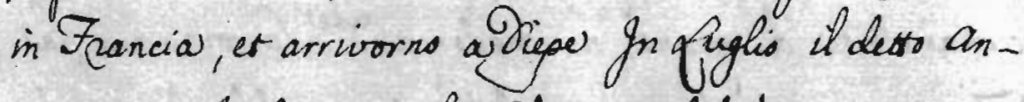

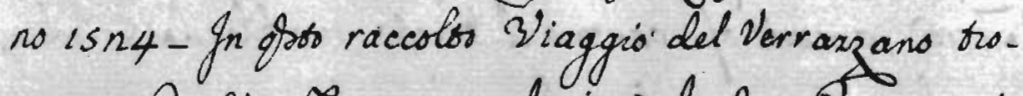
viams la visualione; e condizione di grox laye grof-

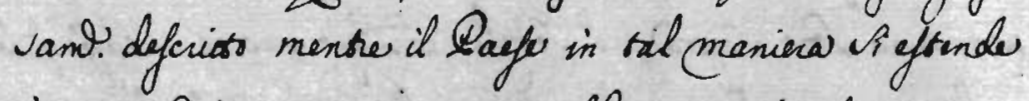

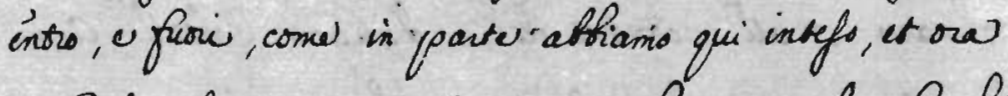
applefos Vensirems; Masicomes Lui in pochi Luoghi fu a terral, non ha' posuts be condifioni de Sagfi,

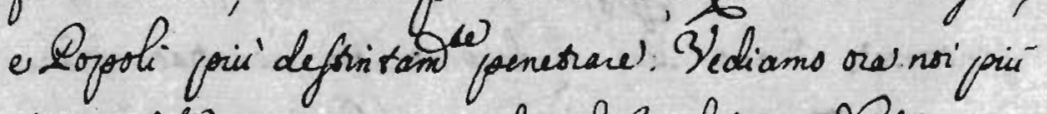

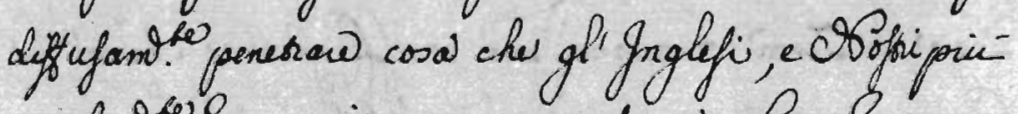

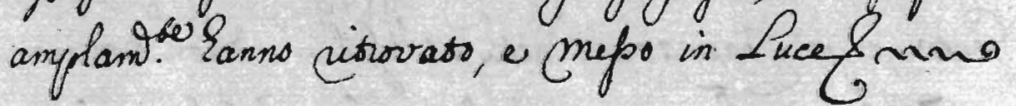


63-66) omitted in $R$. This calls into question Lawrence Wroth's assertion that $R$ represents Verrazzano's own copy of the text. ${ }^{20}$

The transcription of the McGill manuscript which follows includes both the Verrazzano text and the de Laet text. In the former, all var- iations from the published Cèllere, Magliabechian and Cimento manuscripts have been noted, and every fifth line of "McGill" indicated in square brackets. As well, photographs of the McGill manuscript are printed. After more than a century of obscurity, this lost text of Verrazzano is now restored to view.

\section{VERRAZZANO'S COSMOGRAPHY}

Restami gia da narrare a V.S.M. ${ }^{1}$ l'ordine di detta ${ }^{2}$ navigazione circa ${ }^{3} \mathrm{la}^{4}$ cosmografia, come di sopra dissi parten[5]do dalli ${ }^{5}$ prefatti ${ }^{6}$ scopuli che sono' situati nel fine dell' ${ }^{8}$ occidente all' antiquinozio, ${ }^{9}$ e nel meridiano ${ }^{10}$ descritto 11 per le $^{12}$ Insule Fortunate in latitudine ${ }^{13}$ gradi $32^{14}$ dall ${ }^{15}$ equatore del ${ }^{16}$ nostro Emisfero ${ }^{17}$ navigando ${ }^{18}$ all ${ }^{19}$ occidente per fino alla ${ }^{20}$ prima [10]terra trovammo ${ }^{21}$ leghe 1200 , dico $1200,{ }^{22}$ che contengono miglia ${ }^{23} 4800$ computando miglia $4^{24}$ per lega ${ }^{25}$ secondo l'uso maritimo delli navilerii. ${ }^{26}$ Geometrice giustamente ${ }^{27}$ la proporzione $^{28}$ sevisettima ${ }^{29}$ del diametro alla circonferenza ${ }^{30}$ gradi 92 [15] 54164/472733. ${ }^{31}$ Con cio sia che essendo ${ }^{32}$ la corda dell' arco $^{33}$ del massimo circolo ${ }^{34}$ gradi $1146 / 11, e^{35}$ la corda del paralello ${ }^{36}$ gradi 34 della prima terra da noi trovata alla ${ }^{37}$ medesima proporzione ${ }^{38}$ gradi $95223 / 450$ essere si mostra ${ }^{39}$ l'ambito di tutto il circuito $^{40}$ gra[20]di $300313 / 1575^{41}$ che dando per ogni grado come confermano ${ }^{42}$ la maggior ${ }^{43}$ parte di quelli che hanno sperimentato rispondere in terra alla ${ }^{14}$ proporzione ${ }^{45}$ del cielo miglia ${ }^{16} 62$ 1/2 fariano ${ }^{17}$ miglia $1875931 / 126$ quali $^{48}$ repartite $^{49}$ in 360 per veneria per ciascun miglio ${ }^{50} 52$ [25] 1989/9072. ${ }^{51}$ E tanto vale

I. R: V. Mtà.

F: dicta.

F: circha.

R: a la.

R: da li; F: delli.

$\mathrm{R}$ and $\mathrm{F}$ : prefati.

F: son.

F: dello.

R: a li antiqui noto; F: agli antiqui noto.

F: meredian.

R: descripto.

R: la.

R: altitudine di.

R: XXXII.

R: da l'; F: dallo.

$\mathrm{R}$ : nel.

R: Emisperio; F: emisperio.

R: navicammo.

$R$ : a lo.

$\mathrm{R}$ : per insino a la; $\mathrm{F}$ : allo.

F: trovamo.

Omitted in $\mathrm{R}$ and $\mathrm{F}$.

R: migla.

R: quactro.

$R$ and $F$ : legha.

$\mathrm{R}$ : marictimo de navalieri; $\mathrm{F}$ : lo uso marittimo degli.
27. R: geometricie iusta; F: gieometrice giusta.

${ }^{28}$. R: proportione tripla senza; F: proportione tripla.

29. R: sesquiseptima; F: tripla.

30 R: a la circumferentia; F: circunferenza.

${ }^{31}$. F: $925464 / 472733$.

32. R: sendo.

33. R: del archo; F: archo.

34. R: circulo.

35. F: et.

${ }^{36}$. R: pararello di.

${ }^{37}$. R: a la.

${ }^{38}$. $\mathrm{R}$ and $\mathrm{F}$ : proportione.

39. R: monstra.

40. R: el circulo; F: circulo.

${ }^{41}$ R: $300713 / 1515$.

${ }^{42}$. R: confermono.

43. F: maggiore.

44. R: a la.

${ }^{45}$. R and F: proportione.

46. R: migla.

47. R: farieno.

$48^{\circ}$. F: quale.

49. $R$ and $F$ : ripartite.

so. R: ciaschuna migla; $\mathrm{F}$ : ciaschuno.

si. R: 52 989/9072. 
ogni grado di longitudine nel paralello sudetto ${ }^{52}$ di gradi 34 sopra il quale per la retta del meridiano di detti $i^{53}$ scopuli, che stanno in gradi 32 abbiamo calculata ${ }^{54}$ la ragione in questo ${ }^{55}$ che le dette leghe 1200 per retta linea in gradi $34 \mathrm{da}^{56}$ occidente in oriente abbiamo ${ }^{57}$ trovato. Adunque per quelle ${ }^{58}$ a gradi $9254164 / 472733$ e tanto abbiamo ${ }^{59}$ navigato più all' ${ }^{60}$ occidente, che non fù cognito agli antichi ${ }^{61}$ nel detto paralello ${ }^{62}$ di gradi 34 . Questa distanza ${ }^{63}$ a noi fù nota per la longitudi[30]ne con vari ${ }^{64}$ strumenti navigando $^{65}$ senza $^{66}$ eclipsi $^{67}$ lunari ${ }^{68}$ o altro aspetto ${ }^{69}$ per il $1^{10}$ moto solare pigliando" sempre la plenazione ${ }^{72}$ a qual si voglia ora ${ }^{73}$ per la differenza ${ }^{76}$ faceva dall' uno all' altro ${ }^{75}$ orizonte correndo la nave geometrice ${ }^{76}$ ne era noto $\mathrm{l}^{177}$ interval[35]lo dall ${ }^{78}$ uno meridiano ${ }^{79}$ all ${ }^{80}$ altro come in un $^{81}$ libretto tutto amplamente notato ${ }^{82}$ insieme col crescimento del mare in qual si voglia clima a ogni tempo e ${ }^{83}$ ora i $1^{84}$ quale non inutile stimo abbia ${ }^{85}$ a essere a naviganti. ${ }^{86}$ Spero meglio ${ }^{87}$ per la teorica conferirlo a [40]V.S.M. ${ }^{88}$ Mia intenzione ${ }^{89}$ era di pervenire in questa navigazione ${ }^{90}$ al Cathaio ${ }^{91}$ all' estremo ${ }^{92}$ oriente dell' ${ }^{93}$ Asia pensando ${ }^{94}$ trovare tale impedimento di nuova terra quale hò trovata, e se per qualche ragione pensavo quella trovare non senza" ${ }^{95}$ qualche fregio ${ }^{96}$ di penet[45]rare all ${ }^{p 7}$ Oceano Orientale. Essere stimavo ${ }^{98}$ questa opinione $^{99}$ di tutti gl ${ }^{100}$ antichi è stata credendo certamente il ${ }^{101}$ nostro Oceano Orientale ${ }^{102}$ de India uno essere senza interposizione ${ }^{103}$ di terra. Questo afferma A ristotile ${ }^{104}$ argumentando per varie similitudini. La quale opinione [50]è molto contraria a $i^{105}$ moderni e l' esperienza $a^{106}$ falsa. Imperocchè ${ }^{107}$ la terra è stata trovata da quelli ${ }^{108}$ antichi incognita un altro modo ${ }^{109}$ a rispetto di quella ${ }^{100}$ a loro fú

32. R: longitudine nel detto pararello; F: uno detto.

s3. R: del linea recta dal merediano di dicti; F: el merediano.

54. R: habbiamo calculato; F: habbiamo calchulata.

ss. R: impero.

$R: d^{\prime}$.

R: habbiam perverria.

R: quella e; F: e.

R: habbiamo.

R: a lo; F: allo.

R: a li; F: alli antiqui.

$\mathrm{R}$ : decto pararello.

$R$ and $F$ : distantia.

F: varii.

F: navicando.

$\mathrm{R}$ : sanza.

F: eclissi.

R: lunare.

$R$ : aspecto.

F: al.

$\mathrm{R}$ : piglando.

$\mathrm{R}$ and $\mathrm{F}$ : elevatione.

$\mathrm{R}$ : hora.

F: differentia.

R: da l' uno et l'altro.

$\mathrm{R}$ and $\mathrm{F}$ : geometricie.

$R: 10$.

$\mathrm{R}$ : de.

$\mathrm{R}$ and $\mathrm{F}$ : merediano.

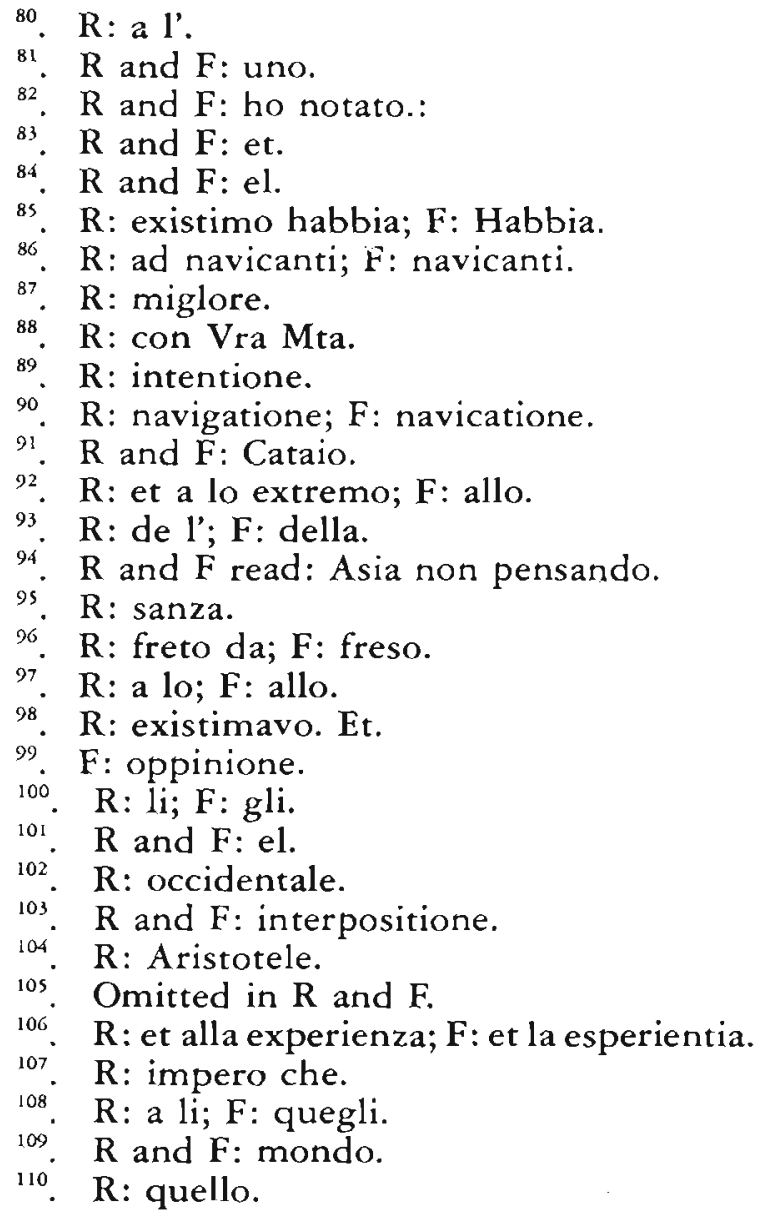


noto. Manifestamente essere si mostra e ${ }^{111}$ di maggiore ${ }^{112}$ della nostra Europa, dell113 Africa, e qua[55]si dell' ${ }^{116}$ Asia. Se rectamente ${ }^{115}$ speculiamo la grandezza ${ }^{116}$ di quella, come sotto brevità ne farò un poco di discorso a V.S.M. ${ }^{117}$ L' equatore distante dal meridiano dall' Insule ${ }^{118}$ Fortunate verso l' occidente gradi $2032060 / 472081^{119} \mathrm{l}^{\prime}$ Ispani ${ }^{120}$ verso l' Austro gradi 54 [60]hanno navigato ${ }^{121}$ dove hanno trovato terra senza ${ }^{122}$ fine. Tornando poi al ${ }^{123}$ settentrione giusta ${ }^{124}$ la detta linea meridionale ${ }^{125}$ correndo il lido ${ }^{126}$ per fino ${ }^{127}$ in otto ${ }^{128}$ gradi propinqui all' equatore più all' occidente participando più al settentrione giusta la detta linea [65]meridionale continovando il lido ${ }^{129}$ per fino in gradi 21 non trovando termine ${ }^{130}$ gradi 89 $29709 / 46782^{13 i}$ hanno navigato, ${ }^{132}$ quali giunti con gradi $2032060 / 46781$ fanno gradi 110 $44830 / 46783,{ }^{133}$ e tanto hanno navigato ${ }^{134}$ dal detto meridiano dall' ${ }^{135}$ Isole Fortunate più all ${ }^{136}$ occiden[70]te, nel paralello gradi 21 dell $^{137}$ altitudine; questa distanza ${ }^{138}$ da noi non è stata sperimentata ${ }^{139}$ per non aver fatta ${ }^{140}$ detta navigazione ${ }^{141}$ potria variare poco più o manco. ${ }^{142}$ Abbiamo ${ }^{143}$ quella calculata ${ }^{146}$ geometrice ${ }^{145}$ per la $[75]$ notizia ${ }^{146}$ di molti navicalieri, ${ }^{147}$ che $1^{1148}$ hanno frequentata. Quali affermano essere legho ${ }^{149} 1600$, giudicando per l' arbitrio ${ }^{150}$ el discorso della ${ }^{151}$ nave secondo la qualità ${ }^{152}$ del vento, per la continova ${ }^{153}$ navigazione. ${ }^{154}$ Spero in breve, ne daremo ${ }^{155}$ ottima ${ }^{156}$ certitudine. Dall'157 altra parte noi in questa nostra ${ }^{158}$ navigazione fatta ${ }^{159}[80]$ per ordine di Vostra S.M. ${ }^{160}$ oltre i gradi $92 \mathrm{che}^{161}$ dal detto meridiano ${ }^{162}$ verso $l^{163}$ occidente dalla ${ }^{164}$ prima terra trovammo ${ }^{165}$ gradi 34 , navigando ${ }^{166}$ leghe 300 , dico leghe 300 , intra ${ }^{167}$ oriente $\mathrm{e}^{168}$ settentrione $^{169}$ leghe 400 quasi all ${ }^{190}$ oriente con-

$111^{\circ}$
$112^{\circ}$
$114^{\circ}$
$115^{\circ}$
$216^{\circ}$
$117^{\circ}$
$118^{\circ}$
$119^{\circ}$
$120^{\circ}$
$121^{\circ}$
$122^{\circ}$
$123^{\circ}$
$124^{\circ}$
$125^{\circ}$
126
$126^{\circ}$
$128^{\circ}$
$129^{\circ}$
$130^{\circ}$

F: maggiore grandezza.

R: de la.

R: de la; F: della.

$F$ : rettamente.

$R$ and $F$ : grandeza.

R: V. Mta.

R: de le isole; F: dalle. McGill omits ultra.

R: 20 32060/4781; F: 20 32060/47281.

R: l'Hispani; F: gli Spani.

F: navicato.

$\mathrm{R}$ : sanza.

R: di.

$\mathrm{R}$ : septemtrione iusta.

$\mathrm{R}$ : meredionale.

R: lito; F: el lito.

$R$ : insino.

R: octo.

F: continuando el lito; not in R.

The phrase "propinqui all' equatore...non trovando termine" (lines 63-66) is omitted in $\mathrm{R}$.

131. R: $892970 / 46781$.

132. F: navicato.

${ }^{133}$. F: 110 et $44830 / 46783$.

134. F: navicato.

135. R: merediano de le.

136. R: a lo; F: allo.

137. R: pararello di...de la; F: della.

138. R: distantia.

139. R: experimentata.
140. R: havere facta; F: avere fatta.

${ }^{141}$. $\mathrm{R}$ and $\mathrm{F}$ : navigatione.

142. F: mancho.

143. R and F: habbiamo.

144. F: calchulata.

${ }^{145}$. R and F: geometricie.

146. R and F: notitia.

147. R: naveleri periti.

148. F: la.

149. R: leghe.

150. R: albitrio.

151. R: de la.

152. R: equalita.

153. F: continua.

154. R: navicatione.

iss. R: hareno.

156. R and F: optima.

157. R: da l'.

158. Omitted in R.

159. R: navicatione facta.

160. R: V. Mta.

161. F: ch'è.

${ }_{162}$. $\mathrm{R}$ and $\mathrm{F}$ : merediano.

163 . F: lo.

${ }^{164}$. R: de la.

165. F: trovamo.

166. R: navicammo; F: navicando.

167. F: infra.

168. R and F: et.

169. R: septemtrione et.

170. R: a l'; F: et allo. 
tinovo, è sito ${ }^{171}$ della terra sia[85]mo pervenuti ${ }^{172}$ infino a gradi $50,{ }^{175}$ lasciando ${ }^{174}$ la terra, che più tempo fa trovorno ${ }^{175} \mathrm{li}^{176}$ Lusitani quali seguirno ${ }^{177}$ più al settentrione ${ }^{178}$ pervenendo sino al circolo artico ${ }^{179}$, e il $^{180}$ fine lasciando ${ }^{181}$ incognito.

Giunta adunque ${ }^{182}$ la latitudine settentrionale ${ }^{183}$ con [90]la meridionale, ${ }^{184}$ videlicet, ${ }^{185}$ gradi 54 con $\mathrm{li}^{186}$ gradi 66 fanno gradi 120 , che tanto contiene ${ }^{187}$ di latitudine $\mathrm{l}^{\prime}$ Africa ${ }^{188}$ con $1^{189}$ Europa, perche giugnendo ${ }^{190} 1^{191}$ estremo $^{192}$ dell' $^{193}$ Europa, che sono i ${ }^{194}$ limiti di Norvegia, ${ }^{199}$ che stanno in gradi 71 con l'estre $[95]_{\mathrm{mo}^{196}}$ dell' ${ }^{197}$ Africa, che il ${ }^{198}$ promontorio, dicano di Buona ${ }^{199}$ Speranza in gradi 35 faranno solo 100 , e se l' equestre di detta terra ${ }^{200}$ in parte corrisponde ${ }^{201}$ al lido marittimo ${ }^{202}$ non è dubbio ${ }^{203}$ di grandezza ${ }^{201} 1^{205}$ Asia ecceda. ${ }^{206}$ In tal forma troviamo $a^{207}$ globo della ${ }^{208}[100]$ terra molto maggiore non hanno tenuto gl' Antichi a repugnanti e mattematici hanno ${ }^{209}$ quella rispetto all ${ }^{210}$ acqua, sia minima, il che per esperienza ${ }^{211} \mathrm{l}^{212}$ opposito veggiamo. ${ }^{213} \mathrm{E}$ quanto all aere ${ }^{214}$ corporale di spazio ${ }^{215}$ non meno la terra che $1^{216}$ ac[105]qua possedere giudichiamo come alla presenza, meglio ${ }^{217}$ spero, e $\operatorname{con}^{218}$ più ragione esperimentare e mostrare a V.S.M. ${ }^{219}$ Tutta questa nova terra ${ }^{220}$ o nuovo mondo, che di sopra abbiamo ${ }^{221}$ narrato, contiene, insieme, congiungendo ${ }^{222}$ all' Asia et

171. R: al lito de la; F: continuo el lito.

$\mathrm{R}$ : pervenendo.

$\mathrm{R}$ : in gradi 54 ; F: per infino...

"Lasciando...", the text of C begins at this point.

$\mathrm{R}$ and $\mathrm{C}$ : trovorono.

R: e; C: i.

R: quale seguirono; C: seguivano.

R: septemtrione; F: septentrione; C: settemtrione.

179. R: per insino al circulo; F: circulo; C: perfino al circulo Articho.

F: 'l.

C: Articho lasando il fine.

R: adonque.

$R$ : septemtrionale; C: settemtrionale.

$\mathrm{R}$ : meredionale.

Omitted in R, F, and C.

$R$ : cio è; $F$ : é.

$\mathrm{R}, \mathrm{F}$ and $\mathrm{C}$ : non tiene.

R: l'Affrica; C: Africha.

F: la.

C: gungendo.

$\mathrm{R}$ and $\mathrm{F}$ : lo.

$R$ : stremo.

R: de la; F: della.

$R$ : e; F: è.

R: Norvegha; F: Norveggia.

R: l' extremo; F: lo.

R: de l'; F: della.

R: che è il; F: che è; $C$ : che è al. Omitted in R, F and C; F: di capo.

$R$ : farieno solo gradi 106 et se il sito ter restre; F: gradi 106...lo sito; C: fariano

gradi 106, et se il sito terrestre.

201

202

$203^{\circ}$

$20-6$.

205

206

$207^{\circ}$

208

$209^{\circ}$

C' hanno voluto; F: gli Antichi repugnantia matematici; C: li antichi et repugnante a matematici ànno voluto.

${ }^{210}$. R and C: a l'; F: alla.

211. R: experientia; F: esperientia; C: isperienza.

212. F: lo.

213. C: oposito veggiamo.

214. R: Et... al Area; F: Et...allo aere; C: Et...all aria.

215. R, F and C: spatio.

$216^{\circ}$. F: la.

${ }^{217}$. R: a la presentia miglore; F: presentia; C: è chome a la presentia miglore.

218. Omitted in $\mathbf{R}, \mathbf{F}$ and $\mathrm{C}$.

219. R: ragioni viste et sperimentate e monstrare a V.Mta; F: experimetare et; C: esperimentar...vostra serenissima maestà.

${ }^{220}$. Omitted in R, F and C.

221. R: habbiamo; $C$ : nuova terra,...e...abiamo.

222. R: non giugnendo a la; F: congiugniendo alla; C: congugnendo. 
Africa, ${ }^{223}$ il che [1 10]sappiamo certo, ${ }^{224}$ potria ${ }^{225}$ giugnere ${ }^{226}$ all ${ }^{227}$ Europa con la Norvegia, e orsia, che sarebbe ${ }^{228}$ falso, secondo ${ }^{229}$ gl' Antichi, quali da promontorio de Cimbi, ${ }^{230}$ quasi tutto il settentrionale dicono ${ }^{231}$ essere stato navicato all ${ }^{232}$ oriente, circuendo circa $^{233}$ il Mare Caspio, e il me[115]desimo, affermano, resterebbe adunque sola $a^{234}$ interclusa ${ }^{235}$ da due ${ }^{236}$ mari silicati dall' oriente et occidentale, e quelle $e^{237}$ due ne chiude ${ }^{238} l^{\prime}$ uno $e^{239} l^{\prime}$ altro perche oltre ai gradi 54 dell' equinoziale ${ }^{240}$ verso $\mathrm{l}^{241}$ austro si stende ${ }^{242}$ all ${ }^{1243}$ oriente per lungo spazio, $\mathrm{e}^{244}$ dal [120]settentrionale ${ }^{245}$ passando $\mathrm{i}^{246}$ gradi 66 segue tornando in ${ }^{247}$ verso $1^{248}$ oriente giugnendo per fino ${ }^{249}$ a gradi 70 . Spero con l' ajuto di V.S.M. ne averemo in breve migliore ${ }^{250}$ certitudine, la quale Dio ${ }^{251}$ onmipotente prosperi a causa veggiamo ottimo ${ }^{252}$ fine di questa nostra [125] cosmografia, che ${ }^{253}$ si adempia la sacra voce dell' Evangelo. ${ }^{254}$ Nella nave Delfina ${ }^{255}$ in Normandia in porto di Diepa a di 8 di Luglio 1524.

$$
\begin{aligned}
& \text { Humilis Servitor }{ }^{256} \\
& \text { Joannes Verrazzanus }
\end{aligned}
$$

\section{LAET'S NIEUIVE WERELDT}

Giovanni da Verrazzano, Fiorentino, fece partenza con carica di commessario di Francesco primo re di Francia, alli 17 di Gennaro 1524 dall isole di Canaria prendendo il suo corso verso occidente con buon vento d' oriente. E nel tempo di giorni 25 - fecero in circa 500 leghe, di poi indirizzorno lor corso d' occidente a settentrione di modo che doppo altri 25 giorni di viaggio scopersero una terra nuova, che giaceva barso, presso l' acqua, e per li fuochi che colà vedevano, giudicorno che dovesse essere abitata, e si missero verso ostro. Veggiorno ben 50 - leghe alle coste per trovare un comodo porto, ma non trovandolo in quell' acque verso ostro, tornarono verso tramontana, dove medesimamente non trovarono porti. Si che si messero verso le coste e mandando il loro coppano verso terra, trovarono molta gente alle rive, o vero gengive, quali
223. F: Africtiá.
224. R: per certeza.
$225^{\circ}$. F: porria.
$220^{\circ}$ C: sapiamo per certeza...gugnere.
$227^{\circ}$ R: a la; F: all.
228 .
229
$\mathrm{R}$ : Norvenga e Rossia, che saria; F:
Norveggia et Rossia; C: Rossia...saria.
F: sicondo.
$230^{\circ}$
231
R: li Antichi...dal...Cimbri; F: gli Antichi... dal...Cimbri; C: li Antichi... dal...Cinbri.
R: el septemtrione dicano; F: septentrio- nale; C: settentrione.
$\mathrm{R}$ : navigato a lo; $\mathrm{F}$ : allo.
F: circha.
Omitted in $\mathrm{R}, \mathrm{F}$ and $\mathrm{C}$.
$C$ : resterebe...interchlusa.
$\mathrm{R}$ : dui.
R: da lo orientale...e quella; F: dallo orientale...et; C: dell' orientale et ociden- tale, et.
$\mathrm{C}$ : dui ne guida; $\mathrm{R}$ and $\mathrm{F}$ omit chiude.
$R$ and $F$ : et.
$\mathrm{R}$ : de lo equinoctiale; F: dello equinotiale;
C: di gradi 54 delle equinotiali.
241. F: lo.
242. C: istende.
${ }^{243}$. R: al; F: allo.
${ }^{244}$ R, F: spatio; C: adds et after spatio.
${ }^{245}$. R: septemtrione; $F$ : septentrionale; $C$ : settentrione.
${ }^{246}$. R: e; F: è.
247. Omitted in $\mathrm{R}, \mathrm{F}$ and $\mathrm{C}$.
248, F: lo.
249. R: per insino; C: gugnendo persino.
${ }^{250}$. R: aiuto di V. Mta ne haremo meglore; F: aiuto... aremo migliore; $\mathrm{C}$ : aiuto vostra serenissima maestà n' aremo...miglore. "ne averemo" omitted in R,F and C.
${ }^{251}$. Omitted in $R, F$ and C. C: Iddio.
252. R: optimo; F: optime; C: vegiamo optima.
${ }^{253}$. R: cosmographia et; $C$ : chonsmografia, et.
${ }^{254}$. R: de lo evangelio; F: bocie dello Evangelio; C: adenpia...del Vangelo.
25s. R: Dalphina a di VIII di luglio M.D.XXIIII; C: in la...Dalfina.
${ }^{256}$. F and C: Humilissimo.
257. R: Janus Verazanus; C: Ioanne Verazano. 
pigliarono la fuga e qualche volta restavano a guardare sopra li Christiani con la bocca aperta. Ma finalmente con segni certificati, che non li saria fatto alcun' inconveniente li mostrarono un buon luogo per venire a terra, et anco li presentarono delle loro vettovaglie. Il popolo andava nudo eccettuato che coprivano le loro vergogne con certe pellicine. Erano di color bruno, neri di capelli, altrimenti belli e proporzionati. Il lido era coperto di fina sabbia, che si estendeva in piccoli muchi, e l' addietro è bello, et alto fondo, e sperso di alberi. Questa terra, che prima scopersero, era sopra l' altezza di 34 gradi verso tramontana. La linea, in una buona e salubre aria, trovarono, che colà l' estate la maggior parte regnavano venti di maestro e ponente, con chiaro e lucido tempo e poca pioggia, e se benne era un lido aperto non trovarono male a starvi con le navi, stante le coste sono libere di scogli. Si partirono di quivi tenendosi lungo le coste, quali trovarono estendersi verso levante, e vederono continovamente molti fuochi a terra, dopo trovarono il paese estendersi verso tramontana il quale si mostrava molto più bello, et ameno, calarono là a terra, e trovarono la gente simile all' antecedente, vederono molte vite, quelli caminavano sopra gli alberi, quelli al loro parere potevano dar buoni frutti in caso fussero state ben coltivate, veleggiarono cosi avanti, secondo il lor parere ben 100 leghe dove trovarono una bella riviera, dove si tirava di nuovo la costa verso oriente, quale proseguirono circa 50 leghe, dove scoprirono un' isola, quale aveva la forma triangolare, giacendo 50 leghe dalla terra ferma piena di montagne, piene e spesse di alberi, e ben abitata, quale nominarono Claudia, 50 leghe più avanti trovarono un buon porto, dove trovorno gente molto buona e trattabile, quale avevano un re, che era vestito con una pelle di cervo artifiziosamente intorta, trovarono fra di loro molto rame rotto, del quale questi salvatici facevano molto conto. Questa terra era giacente sopra l' altezza di gradi $412 / 3$ e si estendeva fra oriente e mezzo giorno, la bocca del porto giaceva aperta verso ponente, largo mezza lega ma di dentro si tirava tra oriente e tramontana 12 leghe, e là veniva più largo e più lungo, e fa un golfo ben largo di 20 leghe, nel quale giacevano cinque piccole isolette, quali erano molto belle e fruttifere, nel mezzo, vel circa dentro di questo porto vi è uno scoglio di pietra, a proposito di fare sopra un castello, o fortezza per difendere il porto. Si partirono di là li 5 Maggio e corsero avanti appresso le coste ben 150 leghe trovando la terra quasi tutta simile se non che era un poco più alta con alcune montagne, la costa si estendeva da 50 leghe verso oriente e doppo di nuovo verso tramontana trovarono colà la gente molto differente dall' antecedente. Mentre sicome quella mostrava esser molto gentile e cortese, era questa molto rustica e salvatica. Siché per nessun messo con questi potevo aver alcun commercio. Erano vestití con pelle di gisi, castori e pelle di pesci marini, e altre pelle pigliano il loro nutrimento quanto lontano possono imaginarsi tra pescare e cacciare, e da alcune radice, che il paese colà da persè produce, non avevano alcuna sorte di grano, ne medemamente. Lavoravano la terra. Ancora osservarono il paese inabile da produre alcun frutto, quando volevano cambiare alcuna cosa venivano loro sopra li scogli, e lasciavano a basso quello volevano, et all' incontro dimandavano coltelli, armi da pescare, e simili robe. Non volevano lasciar venire la gente a terra per qualsivoglia cortesia se li presentarse. Erano però al dispetto loro penetrati qualche lega in terra, tiravano però con le freccie sopra la gente e poi scappavano nelli boschi. Non trovorno in terra cosa di merito o valore, che boschi molto spessi e montagne salvatiche, quale ben mostravano avere in se alcuni minerali, mentre il popolo aveva segnali di rame dall' orecchie pendenti. Partirono di là mettendo il loro corso verso greco longo le coste, quale trovarono più ameno, e con fondo aperto senza alberi con montagne alte fra terra scopersero ben 32 isole tutte giacente vicino a terra, essendo piccole con molte storte, facendo molti buoni porti e canali tra esse isole, e cosi veleggiando avanti verso greco vennero dopo un veleggiamento di 150 leghe, vennero alla terra, o paese avanti scoperto dalli Brittoni, all' altezza di 50 gradi appresso tramontana. La linea d' onde risolsero ritornare in Francia, et arrivorno a Diepe in Luglio ie detto anno 1524.

In questo raccolto viaggio del Verrazzano troviamo la situazione e condizione di questo paese grossamente descritto mentre il paese in tal maniera si estende dentro e fuori come in parte abbiamo qui inteso, et ora appresso sentiremo. Ma si come lui in pochi luoghi fú a terra, non hà potuto le condizioni de paesi e popoli piùdestintamente penetrare. Vediamo ora moi più 
distusamente penetrare cosa che gl' Inglesi e nostri più amplamente hanno ritrovato e messo in luce.

\section{Notes}

The authors would like to thank Ms Lorraine Dubreuil, Map Curator, Department of Rare Books and Special Collections, McGill University Libraries, for bringing this manuscript to their attention.

1. Lawrence C. Wroth, The Voyages of Giovanni da Verrazzano, 1524-1528, (Yale University Press, 1970), 70, n. 21.

2. Ibid., 79.

3. Ibid., 81.

4. Ibid., p. 148 and passim.

5. Alessandro Bacchiani, "Giovanni da Verrazzano e le sue scoperte nell' America settentrionale (1524) secondo l'inedito codice sincrono Cèllere di Roma," Bolletino della società geografica italianna, series IV, vol. X, no. 11 (November 1909), 1274-1323; this was republished with an English translation in A. Bacchiani, "Giovanni da Verrazzano and His Discoveries in North America." Fifteenth Annual Report, 1910, of the American Scenic and Presevation Society (Albany, 1910), Appendix A, 133-202. René Herval provided a French translation, from Bacchiani's text in Giovanni da Verrazzano et les Dieppois à la recherche du Cathay (1524-1528). Étude historique accompagnée d'une traduction intégrale de la célèbre lettre de Verrazzano à François $I^{\text {er }}$... (Rouen et Caen, 1933). Ch.-André Julien republished Herval's translation in Les Français en Amérique pendant la première moitié $d u X V I$ siècle (Paris, 1946), 51-76. Finally, the Italian text accompanied by Susan Tarrow's English translation, is included in Wroth's study of the Cèllere version, 123-143, see note 1.

6. A transcript of this version was presented by George W. Greene to the New York Historical Society and was subsequently edited and published by Joseph G. Cogswell, with an
English translation and introduction, in Collections of the New York Historical Society, 2nd series, vol I (1841), 37-67. The original Italian text was first published by Giovanni Arcangeli, "Lettera di Giovanni da Verrazzano navigatore fiorentino a Francesco I re di Francia sulla scoperta di nuove terre nella costa settentrionale d' America," Archivo Storico Italiano, Appendice, vol. IX (1853), 19-55; and then by Guglielmo Berchet, Raccolta di Documenti e Studi pubblicati dalla $R$. Commissione Colombiana (Rome, 1893), part III, vol. II, 332-341.

7. This copy is in the Department of Rare Books and Special Collections, McGill University Libraries, acc. 2216, G12 R14 folio.

8. Alessandro Bacchiani, Raccolta di Documenti e Studi pubblicati dalla $R$. Commissione Colombiana (Rome, 1893), part III, vol. II, 341-342.

9. Alessandro Bacchiani, "I fratelli da Verrazzano e l' eccidio di una spedizione italofrancese in America (1528)," Bollettino della società geografica italianna, series vi, vol II (Rome, 1925), 374.

10. G.W. Greene, "Life and voyages of Verrazzano," The North American Review, vol xlv, (October, 1837), 308, note.

\section{Ibid.}

12. Napier, Henry Edward," Dictionary of National Biography, vol. xl, 59.

\section{Giuseppe Arcangeli, "Lettera," 20-21.}

14. J.C. Brevoort, Verrazzano the Navigator, or Notes on Giovanni da Verrazzano and on a Planisphere of 1529 illustrating bis American voyage in 1524. A Paper read before the American Geographical Society of New York, November 28th, 1871. (New York, 1874), 33-34. 
15. Bacchiani, Giovanni da Verrazzano e le sue scoperte, 1275.

16. No information on Peter Redpath's acquisition of the Ramusio has been discovered. It was probably acquired for donation to the Library as part of Redpath's plan of long-term support of and acquisition for the Library.

17. The water-mark has not been located in any of the standard catalogues. It consists of the Medici arms surmounted by a royal crown with the letter " $Q$ " underneath. It is $12 \mathrm{~cm}$ in height. The counter mark is an eight-rayed sun with an indistinct image in the centre a chalice? or face? - with the monogram CR underneath. It is $8 \mathrm{~cm}$ in height.

18. Wroth does not indicate this omission in his edition of $\mathbf{R}$; it is clearly a scribal error and an excellent example of a homoeoteleuton. See the transcript for the placement of this and the other variants and errors noted here.

19. Joseph Sabin, A Dictionary of Books relating to America (New York: J. Sabin \& Sons, 1878), vol. $x, 15-16$. The first Dutch edition is 38554 , the second 38555 , the Latin translation 38557, and the French translation [38558].

20. Wroth, Voyages of Giovanni da Verrazzano, $145-146,152$. It should be noted that in this Wroth follows Bacchíani. 